\title{
Thermodynamic incompatibility and complex formation in pectin/caseinate mixtures
}

\author{
Camila F. Rediguieri ${ }^{a}$, Osvaldo de Freitas ${ }^{a}$, M. Paul Lettinga ${ }^{b}$, Remco Tuinier ${ }^{b_{*}}$ \\ Department of Pharmaceutical Sciences, University of São Paulo (FCFRP-USP), \\ Av. Do café s/n, 14040-903, Ribeirão Preto, SP, Brazil ${ }^{a}$ \\ Forschungszentrum Jülich, Institut für Festköperforschung,Weiche Materie, \\ 52425 Jülich, Germany ${ }^{b}$
}

\begin{abstract}
The instability of polysaccharide-protein mixtures occurs either because of thermodynamic incompatibility or complexation. We studied which instability mechanism dominated given the external conditions. Therefore the effect of temperature, $\mathrm{pH}$ and biopolymer concentration on the phase separation of pectin/caseinate mixtures was investigated. At $\mathrm{pH}>6$, thermodynamic incompatibility with spinodal decomposition was observed in pectin/caseinate mixtures resulting in the formation of water-in-water emulsions in intermediate stages of the phase separation process. The demixing rate of these emulsions and appearance of two macroscopic phases (lower phase enriched with caseinate and upper phase with pectin) was retarded when the pectin concentration increased or when the storage temperature decreased, due to a higher viscosity of the mixtures at those conditions.

As the $\mathrm{pH}$ of the mixture was lowered below 6, pectin accumulated in the caseinate-rich phase. Complexation of pectin and caseinate led to the formation of microparticles $(\cong 3 \mu \mathrm{m})$, whose shape depends on the biopolymer concentration ratio and rate of acidification. These pectin/ caseinate particles do not coalesce and are insensitive to salt addition.
\end{abstract}

Keywords: Pectin; caseinate; thermodynamic incompatibility; complexation; microparticles. 


\section{Introduction}

Aqueous mixtures of polysaccharides and proteins are usually unstable above a certain biopolymer concentration and phase separate either because of repulsive (segregative) or attractive (associative) interactions ${ }^{1-4}$. In the first case, also called thermodynamic incompatibility, the two biopolymers do not carry opposite net charges, and after stirring is stopped, the dispersion separates into two phases, each enriched by one of the polymers. In case of complexation, the two biopolymers attract each other because they carry opposite charges and, after demixing, the system consists of a phase containing biopolymer complexes and another phase consisting mainly of solvent. We demonstrate both types of phase instabilities can be found in aqueous mixtures of pectins and caseinates.

Because of the wide applicability of polysaccharide/protein mixtures, the interest in identifying interactions between them has been growing to provide optimum food quality, in terms of texture and stability, and also innovative products in cosmetic and pharmaceutical industries.

Pectin is an anionic linear polysaccharide extracted from citrus peel or apple pomace. Its chemical structure in mainly characterized by D-galacturonic acid units linked together by $\alpha-(1-4)$ glycosidic linkages, occasionally interrupted by $\alpha-(1-2)$ Lrhamnopyranosyl residues. Caseinates are milk proteins whose monomers are complex amphiphilic copolymers with a strong tendency for micellar self-assembly. In milk, caseins are associated with calcium phosphate forming casein micelles (diameter $\cong$ $200 \mathrm{~nm})^{5}$. After removing calcium phosphate, casein micelles dissociate in casein monomers and/or casein submicelles ${ }^{6}$. 
Mixtures of pectin and casein(ate) in aqueous solution are known to be incompatible at $\mathrm{pH} 6.5$ above a certain biopolymer concentration ${ }^{7}$. At lower $\mathrm{pH}$ values, pectin has been used to stabilize acidified dairy beverages due to pectin adsorption onto casein micelles, preventing the aggregation of casein micelles during the acidification process. Much has been published about the interaction of pectin with casein micelles in acidified milk drinks ${ }^{8-11}$, but very few studies were done on pectin/caseinate mixtures at low $\mathrm{pH}$ in calcium-free aqueous solution ${ }^{12,13}$. None of them, however, used pectin concentrations above $1 \mathrm{wt} \%$, because their interest was focused on milk drinks stabilization and that requires just small amounts of pectin.

In this work we study the phase behavior of aqueous pectin plus caseinate mixtures in a wide $\mathrm{pH}$ - and biopolymer concentration range. This enables optimizing the use of pectin/caseinate mixtures in applications (see below). Therefore, a phase diagram was obtained at high $\mathrm{pH}$, where thermodynamic incompatibility takes place, to determine the concentrations where stable and unstable mixtures can be found. Inside the unstable region, water-in-water emulsions were formed and the stability of these emulsions was investigated as a function of biopolymer concentration and storage temperature. At low $\mathrm{pH}$, we investigated the complex formation between pectin and caseinate and the conditions to obtain big spherical particles of the complex. The process to obtain such particles, which is simple and performed in mild conditions, could be used as a microencapsulation technique for pharmaceutical compounds encapsulation or for encapsulation of flavors and odors in food products. The advantage of working with pectin and caseinate is that they are biodegradable polymers with recognized alimentary use and no toxic effects in their utilization are expected. 


\section{Materials and methods}

\subsection{Materials}

High methoxy citrus pectin GENU $^{\circledR}$ type USP-B with $68 \%$ degree of estherification, kindly donated by CP Kelco Limeira - Brazil. CPKelco indicates the molar mass is between 110 and $130 \mathrm{~kg} / \mathrm{mol}$. Commercial bovine milk sodium caseinate was a gift from Kauffmann \& Co., Germany. The caseinate powder contains $92-94 \%$ protein and has a maximum humidity of $12 \%$. Fluorescein-5-isothiocyante (FITC) and rhodamine B were purchased from Sigma-Aldrich, dibutyltin dilaurate and pyridine from Fluka. All the others reagents used in this study were of analytical grade.

\subsection{Methods}

\subsubsection{Casein characterization}

A semi-quantitative characterization of casein was performed using polyacrylamide gel electrophoresis (PAGE) technique, according to the method of

Laemmli $^{14}$ in a discontinuous gel system composed of 5 and $12.5 \%$ acrylamide stacking and running gel, respectively. Sodium caseinate solution at $\mathrm{pH} 7$ was diluted in $0.025 \mathrm{M}$ Tris-HCl buffer $\mathrm{pH} 6.8$ containing $20 \%$ glycerol, $0.02 \%$ bromophenol blue and $0.2 \mathrm{M}$ DTT (dithiothreitol) and boiled for 5 minutes at $100^{\circ} \mathrm{C}$. Samples with 40, 20 and $10 \mathrm{mg}$ protein were loaded into a vertical electrophoresis cell (Hoefer SE 600, Amersham Biosciences) at $12 \mathrm{~mA}$ and ran until the dye front reached the bottom of the gel. The electrode buffer was $0.025 \mathrm{M}$ Tris- $\mathrm{HCl} \mathrm{pH} 6.8,0.192 \mathrm{M}$ Glycine and $0.1 \% \mathrm{SDS}$. The gel 
was stained with a Coomassie brilliant blue G-250. First it was incubated for 1 hour in a $40 \%(\mathrm{v} / \mathrm{v})$ ethanol and $10 \%(\mathrm{v} / \mathrm{v})$ acetic acid solution and then immersed for 12 hours in $0.1 \% \mathrm{w} / \mathrm{v}$ Coomassie G-250, $2 \% \mathrm{w} / \mathrm{v}$ phosphoric acid, $10 \% \mathrm{w} / \mathrm{v}$ ammonium sulfate, $20 \%$ $\mathrm{v} / \mathrm{v}$ methanol. For destaining, $25 \% \mathrm{v} / \mathrm{v}$ methanol was used and the images were acquired with ImageScanner II (Amersham Biosciences).

\subsubsection{Labeling}

To visualize pectin and caseinate with confocal scanning light microscopy (CLSM), they were labeled with fluorescent dyes. Pectin was labeled covalently with FITC according to the procedure described by Tromp and co-workers ${ }^{15}$. Pectin $(1 \mathrm{~g})$, FITC $(0.14 \mathrm{~g})$, pyridine $(400 \mu \mathrm{l})$, and dibutyltin dilaurate $(80 \mu \mathrm{l})$ were dissolved in 100 $\mathrm{mL}$ of DMSO. The reaction mixture was heated for $4 \mathrm{~h}$ at $100^{\circ} \mathrm{C}$, poured into isopropyl alcohol $(400 \mathrm{ml})$ and centrifuged for $55 \mathrm{~min}$ at $9500 \mathrm{~g}$ at $20^{\circ} \mathrm{C}$. Subsequently, the polysaccharide sample was redissolved in $200 \mathrm{ml}$ of hot water and dialyzed against $\mathrm{NaCl}$ $0.1 \mathrm{M}$ solution $(4 \times 41)$ and distilled water $(2 \times 41)$. In this manner an excess of unbound dye and salt was removed. After that, the sample was freeze-dried.

To calculate the number of FITC molecules per galacturonic acid molecule, first a calibration curve for FITC was constructed. An amount of $10 \mathrm{mg}$ FITC was dissolved in $100 \mathrm{~mL}$ DMSO. Dilutions of this solution in $0.01 \mathrm{M}$ phosphate buffer was used to construct the calibration curve, measured at a wavelength of $490 \mathrm{~nm}$, from which the molar extinction coefficient $(\varepsilon)$ was calculated, giving $\varepsilon=73789 \mathrm{~L} \mathrm{~mol}^{-1} \mathrm{~cm}^{-1}$. To determine the number of dyes per pectin monomer, we dissolved a known amount of FITC-pectin in buffer and detected the absorption of the solution at $490 \mathrm{~nm}$. The dye 
concentration then follows from absorption plus obtained extinction coefficient. We found that each milligram of labeled pectin contained $20.45 \mu \mathrm{g}$ of FITC. Considering that pectin mainly consists of galacturonic acid monomers with a molar mass close to 200 $\mathrm{g} / \mathrm{mol}$, the number of FITC molecules/galacturonic acid molecule was estimated to be about 0.01 . The fact that we have only one dye molecule per hundred sugar monomers and that the phase behavior does not change with the small amount of labeled pectin used implies that we cannot expect large affects of labeled pectin on the physical properties.

Caseinate was labeled non-covalently with Rhodamine B by adding one drop of a $0.02 \%$ solution to $10 \mathrm{ml}$ biopolymer mixture.

\subsubsection{Phase diagram determination}

Pectin and sodium caseinate were dispersed separately in $0.01 \mathrm{M}$ phosphate buffer pH 7.0 containing sodium azide $\left(\mathrm{NaN}_{3}\right) 0.03 \mathrm{wt} \%$ to prevent bacterial contamination. Stock solutions of $1.76 \mathrm{wt} \%$ pectin and $10 \mathrm{wt} \%$ caseinate were used to prepare mixtures as to establish the phase diagram. Equal volumes of the diluted solutions were mixed and the $\mathrm{pH}$ was adjusted to 7.0 with $4 \mathrm{M} \mathrm{NaOH}$. One gram of the mixtures was weighed in glass tubes and left overnight to phase separate at $45^{\circ} \mathrm{C}$. The concentrations of the biopolymers in both phases were determined spectrophotometrically. Two series of samples were prepared, one containing labeled pectin (50 mg of pectin-FITC/g unlabeled pectin) and another with only unlabeled pectin for caseinate quantification. No difference on the phase volumes was observed between the labeled and unlabeled samples. To measure pectin and caseinate concentrations, calibration curves for the two polymers were constructed at $\mathrm{pH}$ 7.0. The absorbance values were taken at a wavelength of $490 \mathrm{~nm}$ 
for pectin-FITC $\left(\varepsilon=0.21 \mathrm{~L} \mathrm{~g}^{-1} \mathrm{~cm}^{-1}\right)$. To quantify the amount of caseinate, the Bradford reagent was used ${ }^{16}$ and the absorbance values were taken at $595 \mathrm{~nm}, \quad$ where $\varepsilon=$ $0.63 \mathrm{~L} \mathrm{~g}^{-1} \mathrm{~cm}^{-1}$.

The most upper tie-line in the phase diagram was obtained from solutions of 6 $\mathrm{wt} \%$ pectin and $6 \mathrm{wt} \%$ caseinate. In this case, the phase separation took a long time and, therefore, $1 \mathrm{~g}$ of the mixtures were weighed in small vials and centrifuged in a Microfuge 18 centrifuge (Beckman Coulter) at $2296 \mathrm{~g}$ for 1 hour. Also, as the lower phase in this sample was very viscous and difficult to pipette, the concentration in that phase was obtained by subtracting the amount of the biopolymers (pectin and caseinate) in the upper phase from the total amount. The volumes were determined by marking the interface position on the outside of the tubes and, after emptying, by weighing the amount of water corresponding to the marked volumes.

\subsubsection{Effect of $\mathrm{pH}$ on pectin/caseinate mixtures}

Equal volumes of pectin $6 \mathrm{wt} \%$ and caseinate $6 \mathrm{wt} \%$ solutions were mixed and the $\mathrm{pH}$ was adjusted to 8.0 with $4 \mathrm{M} \mathrm{NaOH}$. Under magnetic stirring, the mixture was acidified with $1 \mathrm{M}$ citric acid to various $\mathrm{pH}$ values between 8 and 3 . At each $\mathrm{pH}, 5 \mathrm{~g}$ of the mixtures were collected and centrifuged in a TJ-25 Centrifuge (Beckman Coulter) at $5000 \mathrm{~g}$ for 3 hours. Again, two series of samples were prepared for quantification of pectin and caseinate in the upper phases (according to item 2.2.3). Calibration curves were established at every $\mathrm{pH}$. The concentrations in the lower phase were obtained by subtracting the amount of the biopolymers in the upper phase from the total amount present in the mixture. The volumes were measured as described also in section 2.2.3. 


\subsubsection{Stability}

The stability of pectin/caseinate mixtures at $\mathrm{pH} 7.0$ with different polymer ratios was studied at $5,15,30$ and $45^{\circ} \mathrm{C}$. One gram of each mixture was weighed in glass tubes and closed to avoid solvent evaporation. The tubes were kept in the thermostatic bath or fridge for one week. The samples were checked every 24 hours. When the meniscus between the two phases appeared, the sample was considered demixed.

\subsubsection{Rheology}

Rheological experiments of concentrated samples were performed using a rheometer Rheometric Scientific (Ares) in the controlled strain mode with Couette geometry (bob 32mm and cup $34 \mathrm{~mm}$ ). The measurements were made at 5, 15, 30 and $45^{\circ} \mathrm{C}$. A solvent trap was used to avoid evaporation.

The intrinsic viscosity of pectin was determined at $25^{\circ} \mathrm{C}$ by using an ordinary Ostwald viscosimeter. An amount of $15 \mathrm{~mL}$ of each pectin solution, prepared in a phosphate buffer with $\mathrm{pH} \mathrm{7,} \mathrm{was} \mathrm{poured} \mathrm{into} \mathrm{the} \mathrm{viscosimeter.} \mathrm{The} \mathrm{flow} \mathrm{time} \mathrm{of} \mathrm{the}$ samples was measured at different concentrations $(0.25,0.5,0.75,1,1.5,2 \mathrm{wt} \%)$. The dynamic viscosity $\eta$ follows from the flow time multiplied with the density. The density ( $\rho)$ was measured using a pycnometer.

\subsubsection{Microscopic observations}

Transmission microscopy and CSLM observations were carried out on a Zeiss Axioplan 2 with $40 \times$ oil immersion objective lens. The scanning head was a multi-point 
confocal scanner VTinfinity from VisiTech. The excitation lengths for Rhodamine and FITC were 568 and $488 \mathrm{~nm}$ respectively. Emission maxima were detected at wavelengths of 625 and $518 \mathrm{~nm}$.

The microscopic observations of the phase separation were done on a Zeiss Axioplan 2 with $10 \times$ objective lens. Samples containing Rhodamine were mixed in a vortex and $50 \mu \mathrm{L}$ was immediately pipetted on a glass slide. With a spatula the samples were sheared continuously until the sample was well-mixed and, immediately after cessation of mixing, images were taken sequentially.

Image treatment was done with home-written IDL programs, using the standard IDL Fast-Fourier Transform routine. 


\section{Results and discussion}

\subsection{Casein characterization}

The PAGE traces for caseinate solution, shown in Figure 1, are very similar to already reported bovine casein PAGE profiles $^{17-19}$. Two major bands appeared after protein separation, which correspond to $\alpha_{\mathrm{s}}$-casein (approximately $32 \mathrm{kDa}$ ) and $\beta$-casein (approx. $29 \mathrm{kDa}$ ). The amounts of these two fractions were similar. The third fraction, $\kappa-$ casein, appears as a thin and smooth trace (approx. $27 \mathrm{kDa}$ ), indicating a smaller amount. This result agrees with the ratio $4: 1: 4: 1.3$ of the fractions $\alpha_{\mathrm{s} 1}: \alpha_{\mathrm{s} 2}: \beta: \kappa$, generally found for bovine caseins ${ }^{6}$.

\subsection{Phase diagram}

When pectin and caseinate are brought together in aqueous solution the mixture may phase separate into two phases: a lower phase containing mainly protein and an upper phase enriched in polysaccharide. In order to determine the phase coexistence concentrations at which pectin/caseinate mixtures undergo phase separation we constructed a phase diagram, shown in Figure 2, at $\mathrm{pH}$ 7. The solid curve corresponds to the binodal, which distinguishes the compatible (one-phase) region from the incompatible (two-phase) region in the phase diagram. Below the binodal, pectin/caseinate mixtures are stable. As the concentration of the biopolymers is raised above the binodal, the system starts to phase separate. The compatible region is, therefore, small compared to the incompatible one, as expected for polysaccharide/protein mixtures ${ }^{20}$ and according to previous work of Antonov and co-workers ${ }^{7}$. 
In the phase diagram the concentrations of the coexisting phases after phase separation are linked by tie-lines. As the mixture is diluted, while taking care the volumes of the two coexisting phases remain approximately equal, the tie-lines become shorter until the coexisting phase compositions merge in the critical point. At relatively high pectin concentrations along a tie-line, the system is microscopically characterized by droplets of caseinate (dark domains) in a continuous pectin phase (light domains) in Figure 2. If we take the opposite direction towards higher caseinate concentrations, there is a phase inversion and pectin droplets become surrounded by a continuous phase enriched in caseinate. At intermediate positions along a tie-line, where the volume fractions of both phases are similar, a bicontinuous system is observed.

A mixture of caseinate and pectin may be considered as a colloid-polymer mixture in a common solvent. At the temperature studied, caseinates (mainly $\beta$ - and $\alpha$ caseins) form spherical micelles with a diameter of $30 \mathrm{~nm}^{21}$. Pectin molecules in aqueous solution can be regarded as polymer chains. In case polymers (pectin) do not adsorb onto spherical particles (micellar caseinates), the non-adsorbing polymers are depleted from the regions surrounding the spheres. Such regions are called depletion zones ${ }^{22,23}$. When the protein particles approach each other sufficiently close, the depletion zones overlap. Thus, the distance between the two spheres is smaller than the diameter of the polymer particles and therefore no polymers can enter the gap between them. Such a lack of polymers leads to an osmotic pressure imbalance that causes an effective attraction between the spheres. If this attraction is sufficiently strong, the system will phase separate; the spheres and non-adsorbing polymers are thermodynamically incompatible. Thermodynamically, the driving force is purely entropic and, therefore, no temperature 
dependence of the phase coexistence curve is expected in this case. As demonstrated in Figure 3, mixtures incubated at three different temperatures $\left(45,20\right.$ and $\left.5^{\circ} \mathrm{C}\right)$ had similar results for the coexisting phase concentrations after phase separation.

In Figure 4 we compared the experimental coexistence curve for pectin/caseinate mixtures at $\mathrm{pH} 7$ (panel A) to a theoretical prediction ${ }^{24}$ for mixtures of hard spheres plus polymer chains with excluded volume interaction between the segments (panel B). The theoretical phase diagram is only a function of $q=R_{g} / a$, with hard sphere radius $a$ and the radius of gyration of the polymer chains $R_{\mathrm{g}}$. Caseinates are mainly present as spherical micelles with a radius $a \approx 15 \mathrm{~nm}^{21}$. For the pectin polymers we have no information of the size of the chains. Therefore we match the critical point of the theoretical prediction to the experimental critical point. The critical caseinate concentration is close to $40 \mathrm{~g} / \mathrm{L}$, see Figure 2. The volume fraction $\phi$ of the spherical micelles can be converted to the caseinate concentration by using the voluminosity of caseinate micelles, being close to 4 $\mathrm{mL} / \mathrm{g}^{21}$. We therefore set $q=R_{g} / a=1.5$ in the calculations since this corresponds to a critical volume fraction at $\phi=0.17$. The polymer concentration at the critical point is found in terms of $\mathrm{c} / \mathrm{c}^{*}$, where $\mathrm{c}^{*}$ is the overlap concentration. Theoretically, we find $\mathrm{c} / \mathrm{c}^{*}$ $\approx 2$ at the critical point for $q=1.5$. We thus assure $\mathrm{c} / \mathrm{c}^{*}=2$ corresponds to the pectin concentration at the critical point determined experimentally, which is $5.6 \mathrm{~g} / \mathrm{L}$. This means $\mathrm{c}^{*}$ would be close to $2.7 \mathrm{~g} / \mathrm{L}$. Since $\mathrm{c}^{*} \approx 1 /[\eta]$, where $[\eta]$ is the intrinsic viscosity, the intrinsic viscosity would be of the order of $0.4 \mathrm{~L} / \mathrm{g}$.

We measured the viscosity of the pectins in solution we used in the dilute regime. In Fig. 5 we plot the specific viscosity over the pectin concentration as a function of 
pectin concentration. The intrinsic viscosity $[\eta]$ follows from the intercept and is 0.27 $\mathrm{L} / \mathrm{g}$, close to the result of $0.3 \mathrm{~L} / \mathrm{g} .{ }^{25}$

We plot the theoretical phase coexistence curve in Figure 3 (panel B), including a few representative tie-lines. The resulting theoretical phase diagram is very similar to the experimental phase diagram, which supports the idea that the phase behavior is only driven by entropic forces. It is noted that a prediction for hard spheres plus ideal (noninteracting) chains looks differently; the binodal then looks more like a $1 / x$ decaying function. The main difference between panels $\mathrm{A}$ and $\mathrm{B}$ is that the experimental tie-lines depend more strongly on caseinate concentrations.

\subsection{Kinetics of phase separation}

The phase separation process can usually be described in terms of two mechanisms: a) nucleation and growth, for mixtures with concentrations in the metastable region of the phase diagram, between the binodal and spinodal and b) spinodal decomposition, for mixtures in the unstable region above the spinodal curve. Close to the critical point, where the binodal and spinodal meet, an increase in polymer concentrations directly leads to a transition from the stable region to the spinodal region, without crossing the meta-stable region. To visualize spinodal decomposition in pectin/caseinate mixtures, samples close to the critical point were investigated with equal volumes of the two phases, but also with $75 \%$ pectin phase $/ 25 \%$ caseinate phase and $25 \%$ pectin phase $/ 75 \%$ caseinate phase along the same tie-line.

As can be seen in Figure 6, after mixing was stopped structure formation was observed for the $50 \% / 50 \%$ sample which is characteristic of spinodal decomposition. 
From the interconnected structure seen in the microscopic images (left side of the insets in Figure 6A) we see the formation of the typical interconnected structure, and the Fourier transform of the images shows the appearance of a ring structure, which is typical for spinodally decomposing samples ${ }^{26,27}$. The peak intensity is found at the typical wavelength which we denoted as $\mathrm{Q}_{\max }$. In Figure $6 \mathrm{~B}$, the characteristic length scale $1 / \mathrm{Q}_{\max }$ is plotted as a function of time after cessation of mixing. Shortly after mixing $1 / Q_{\max }$ is apparently nearly constant, which is sometimes observed in relatively viscous demixing polysaccharide-protein mixtures ${ }^{27}$. Above $\mathrm{t} \sim 1 \mathrm{~s}, 1 / \mathrm{Q}_{\max }$ starts to increase significantly with time. In the regime around $\mathrm{t} \sim 2 \mathrm{~s}$, $\mathrm{Q}_{\max }$ scales as $1 / \mathrm{Q}_{\max } \sim \mathrm{t}^{0.52}$. For $\mathrm{t} \sim$ $10 \mathrm{~s}, \mathrm{Q}_{\max }$ scales as $1 / \mathrm{Q}_{\max } \sim \mathrm{t}^{0.76}$. These scaling exponentials are at variance with theoretical estimates for spinodal decomposition of $1 / \mathrm{Q}_{\max } \sim \mathrm{t}^{1 / 3}$ and $1 / \mathrm{Q}_{\max } \sim \mathrm{t}^{1}$ for concentrated mixtures where hydrodynamics dominates, which are both found experimentally ${ }^{28,29}$.

For the samples $75 \% / 25 \%$ and $25 \% / 75 \%$, even after high shear rates, the droplets did not disappear. According to Onuki ${ }^{30}$, domains that are more viscous than the continuous phase tend to be closed-shaped because the continuous phase is more easily deformed into extended shapes. Therefore, for those samples, the spinodal decomposition in the very early stages could not be visualized.

\subsection{Stability}

Pectin and caseinate concentrations strongly affect the phase separation rate of the biopolymer mixture, since the concentration of biopolymers influences both the thermodynamic forces in the mixture and the viscosity of the system. High polymer 
concentrations increase the thermodynamic incompatibility that induces the phase separation but, on the other hand, increasing the viscosity decelerates the demixing process. Thus, the combination of both factors determines the demixing rate. If the biopolymer concentration increases and the phase separation rate slows down this is due to the fact that viscous forces dominate, while thermodynamic forces dominate if the phase separation rate goes up.

Figure 7 indicates the time required for macroscopic phase separation of some pectin/caseinate mixtures at four temperatures $5,15,30$ and $45^{\circ} \mathrm{C}$. The samples were checked every 24 hours for one week. It is clear phase separation is retarded when the polymer concentration in the mixture increases (compare mixtures 20/20, 30/30, and $40 / 40 \mathrm{~g} / \mathrm{l})$. Indeed, when the caseinate concentration is kept constant and the pectin concentration increases, the demixing process is also retarded (see caseinate $10 \mathrm{~g} / 1$ and 40 $\mathrm{g} / \mathrm{l})$. In these cases the increasing viscosity of the mixtures is the dominating factor determining the demixing rate. However, when the pectin concentration is kept constant and the caseinate concentration is increased, the demixing process either slows down (see pectin $20 \mathrm{~g} / 1$ at $15^{\circ} \mathrm{C}$ ) or accelerates (pectin $30 \mathrm{~g} / 1$ at 30 and $45^{\circ} \mathrm{C}$ ). In the last case the thermodynamic forces dominate with respect to the rheological properties of the system.

In the late stage of spinodal decomposition, phase separation is governed by diffusion of droplets. Because of the high viscosity of concentrated polymer solutions, diffusivity of the droplets in the system decreases with increasing concentration. The low diffusivity consequently reduces the collisions between the droplets. Hence, coalescence slows down and the demixing process may even become arrested. The effect of gelation 
on coarsening prevention has been reported in systems containing gelatin ${ }^{31-34}$ and HPMC 35

Besides biopolymer concentration, another factor that influences the viscosity, and consequently the demixing rate, is temperature. At higher temperatures the demixing process for pectin/caseinate mixtures occurs faster. At 45 and $30^{\circ} \mathrm{C}$ the macroscopic phase separation took place within 6 days. At 15 and $5^{\circ} \mathrm{C}$ most of the samples were still stable after 1 week.

Figure $8 \mathrm{~A}$ shows the viscosity of a pectin/caseinate mixture as a function of temperature. A mixture with a composition of $30 / 30 \mathrm{~g} / 1$ was chosen for this experiment. The viscosities plotted were viscosities measured at a shear rate of $0.1 \mathrm{~s}^{-1}$. As expected, the viscosity of the mixture increases with decreasing temperature. This change in viscosity is strongly correlated with the demixing duration (figure 8B). Although viscosity is not the only factor that dictates the phase separation rate it is clear that is does play an important role. Another factor that in general affects the phase separation rate is the interfacial tension ${ }^{28}$, which probably hardly changes with temperature. Depending on the storage temperature, these water-in-water mixtures can be stable for approximately a month.

\subsection{Effect of $\mathrm{pH}$ on pectin/caseinate mixtures}

An interesting finding was made when the $\mathrm{pH}$ of the mixture was lowered to 5 . Although the viscosity of the mixture was lower at that $\mathrm{pH}$ than at $\mathrm{pH} 7$ (figure 8A), the macroscopic phase separation took much longer (more than a week) at $45{ }^{\circ} \mathrm{C}$. This 
suggests that the driving force for a segregative phase separation becomes weaker at lower $\mathrm{pH}$ values and thermodynamic incompatibility is suppressed. If complexation occurs, two phases would be seen only after sedimentation of the complexes, and this could take a long time depending on the density and size of the complex particles.

As to investigate what happens at lower $\mathrm{pH}$, the concentration of the biopolymers in both phases was measured for a 30/30 g/l mixture at different $\mathrm{pH}$ values. First, a mixture was prepared with $\mathrm{pH} 8$ and then, by adding citric acid, the $\mathrm{pH}$ was brought down until the desired $\mathrm{pH}$ was reached. Figure 9A shows the relative concentration of pectin in both upper and lower phases compared to the initial concentration as a function of $\mathrm{pH}$.

At high $\mathrm{pH}$ values the concentration of pectin in the upper phase is higher than the concentration in the original mixture. In the lower phase, only small amounts of the polysaccharide are found. This reflects a phase separation driven by segregative interactions. As the $\mathrm{pH}$ is lowered, the concentration of pectin in the upper phase decreases while the concentration increases in the lower phase. Because the lower phase volume is much smaller than the upper phase volume (it can be 10 times smaller depending on the $\mathrm{pH}$ ), the concentration in the lower phase increases strongly with decreasing $\mathrm{pH}$. The crossover point at $\mathrm{pH} 5.5$ indicates that at this $\mathrm{pH}$ the concentration of pectin is equal in both phases and that below this point pectin becomes more concentrated in the lower phase. In contrary to pectin, caseinate remained more concentrated in the lower phase independent of the $\mathrm{pH}$. These results suggest that pectin and caseinate concentrate in a single phase below $\mathrm{pH} 5.5$, which is characteristic for complexation. 
The reason why at $\mathrm{pH}>6$ pectin/caseinate mixtures phase separate is that the polymers repel each other. Under those conditions both pectin, with $\mathrm{pK}_{\mathrm{a}}$ of carboxylic groups close to 3 , and caseinate, with $\mathrm{pI} \cong 4.6$, carry negative charges, which makes repulsive electrostatic and excluded volume interactions dominant. In that case, incompatibility takes place driven by depletion forces. However, as the $\mathrm{pH}$ of the mixture is lowered, caseinate becomes less negative. Even though the protein carries a net negative charge above the $\mathrm{pI}$, the presence of regions on the caseinate molecules that are positively charged even at high $\mathrm{pH}$ can attract the negatively charged pectins. This attraction becomes stronger with decreasing $\mathrm{pH}$ below the isoelectric point, when the amphoteric caseinate becomes positively charged.

Figure 10 shows images of $30 / 30 \mathrm{~g} / 1$ mixture in transmission and confocal microscopy. At pH 7 and 6 the mixtures (rows A and B) consisted of caseinate-rich droplets in a pectin-rich continuous phase. At pH 5.5 confocal contrast is negligible, which means that the concentration of pectin in the droplets and in the continuous phase becomes similar. This agrees with the results of figure 9A where the upper phase concentration curve meets the lower phase close to $\mathrm{pH}$ 5.5. Further lowering of the $\mathrm{pH}$ ( $\mathrm{pH}$ 5) pushes more pectin into the droplets and they become brighter than the outside medium. The 'migration' of pectin from the continuous phase to the particles explains the lower viscosity of the mixture at $\mathrm{pH} 5$ compared with $\mathrm{pH} 7$ (figure 8A).

Images of the lower phases after phase separation (figure 10C) were also investigated. At $\mathrm{pH}$ 7, the lower phase was mainly caseinate-enriched with some small pectin-rich droplets. At $\mathrm{pH} 6$, the lower phase was not a continuous phase, but rather caseinate-rich particles interconnected by pectin-rich areas, indicating that coalescence 
was prevented. At $\mathrm{pH} 5.5$ contrast nearly vanishes, while particles become distinguishable again at $\mathrm{pH}$ 5. Figures 9B,C show the lower phase content at different $\mathrm{pH}$ values. In panel B, we see that the mass ratio pectin/caseinate increases with decreasing $\mathrm{pH}$. At $\mathrm{pH}$, although pectin is not concentrated in the particles, the amount in the lower phase increases because it stays entrapped between the particles (figure 10C). This causes an increase in the volume of the lower phase which explains the reduction of total biopolymer concentration in the lower phase at that $\mathrm{pH}$ (figure 9C). Visual observations of the mixtures also indicates that complexation occurs at $\mathrm{pH}$ values smaller than 6 . Between $\mathrm{pH} 8$ and 6.5, the mixture was transparent and so were the two phases after demixing. Starting from $\mathrm{pH} 6$, the mixture became more turbid and at 5 it was opalescent-white, indicating the presence of insoluble matter (complexes). At this point, the bottom phase after centrifugation was solid and not liquid as for high $\mathrm{pH}-$ mixtures.

The slow acidification of polysaccharide/protein mixtures from a $\mathrm{pH}$ where no associative interaction occurs has been performed before by using glucono- $\delta$-lactone (GDL) to study electrostatic adsorption of pectins onto casein micelles in milk ${ }^{10}$, and electrostatic complexation between $\beta$-lactoglobulin and xanthan ${ }^{36}, \beta$-lactoglobulin and pectins $^{37}$, and $\beta$-lactoglobulin and acacia gum ${ }^{38}$. However, the use of xanthan or pectins as polyanionic biopolymers did not induce the formation of coacervates, but resulted in rather small ${ }^{10}$ or fractal aggregates with fractal dimensions in the range $1.7-2.5^{37}$. Liquid droplets or vesicles were observed only with acacia gum ${ }^{39,40}$.

In our case, spherical particles with $\cong 3 \mu \mathrm{m}$ diameter were obtained after lowering the $\mathrm{pH}$ slowly ( $\cong 1$ drop of citric acid/minute) from 7 to 5 , for mixtures with high pectin concentrations (figure 11, situation A). When the $\mathrm{pH}$ was lowered fast (a single drop of 
citric acid/second), the particles lost sphericity. It seems that pectin needs time to diffuse into the droplets and adsorb onto micellar caseinates and stabilize them. When the acidification is performed rapidly, not all micelles become stabilized by pectin molecules and some of them aggregate, resulting in non-spherical particles. This also happens when the concentration of pectin in the mixture is reduced (figure 11, situations B, C). In such cases, the amount of pectin is insufficient to stabilize all caseinate micelles and, even with slow acidification, the particles become non-spherical. For samples with volume fractions of $50 \%$ (situation $\mathrm{C}$ in figure 11 ) and $25 \%$ pectin/ $75 \%$ caseinate (not shown), the concentration of pectin was, compared to caseinate concentration, low to such a degree that, at $\mathrm{pH} 5$, pectin was still concentrated in the continuous phase (the particles were dark). Because the amount of pectin available to complex with caseinate was so small, caseinates needed to have more positively charged groups $(\mathrm{pH}$ below the isoelectric point) to attract more pectin, so that the particles would become more concentrated in pectin than the continuous phase was. It is important to mention that this complexation in pectin/caseinate mixtures is reversible. As the $\mathrm{pH}$ is increased again, the pectin molecules migrate from the particles to the bulk phase and the droplets become flexible, start coalescing as happens at high $\mathrm{pH}$ values.

Despite the $\mathrm{pH}$ sensitivity, pectin/caseinate complexes are found to be insensitive to salt addition. This contrasts with the behavior of other studies on polysaccharide/protein complexes ${ }^{41,42}$. Even at high ionic strengths $(100 \mathrm{mM} \mathrm{NaCl})$ the particles did not change into soluble complexes or to a segregation of pectin and caseinate. It is a well-known phenomenon that the microions present in the solution screen the charges of the polymers and thus reduce the range of their associative 
interactions $^{2}$. In pectin/caseinate complexes, maybe because of the high biopolymer concentration used (total $60 \mathrm{~g} / 1$ ), the amount of microions was below that required for complexation suppression.

\section{Conclusions}

In this paper we have shown that $\mathrm{pH}$ and biopolymer concentration play an important role in the phase stability of pectin/caseinate mixtures. A phase diagram constructed at $\mathrm{pH} 7$, where both polymers are negatively charged, reveals the mixture is stable only at small biopolymer concentrations. Higher concentrations lead to phase separation, due to thermodynamic incompatibility, and formation of water-in-water emulsion. We could confirm thermodynamic incompatibility occurs at $\mathrm{pH}>6$, because the phase line in that regime is insensitive to temperature and the observed phase diagram could be rationalized by comparing with theory for a dispersion of hard spheres (mimicking micellar caseinates) plus polymer chains (mimicking pectin polymers) in solution with excluded volume interaction between the segments. Spinodal decomposition was observed in the early stages of the phase separation. The phase separation rate slows down as the concentration of pectin in the system is increased, due to the higher viscosity of those mixtures. Because viscosity changes with temperature, the stability of the emulsions is also dependent on the storage temperature.

When the $\mathrm{pH}$ of the incompatible mixture is lowered below 6, complexation takes place, because caseinate then becomes significantly positively charged and attracts the negatively charged pectin molecules, resulting in formation of big pectin/caseinate 
particles that are resistant to high ionic strengths. These particles are more spherical at high pectin concentrations and when the acidification is performed slowly, because of better stabilization of micellar caseinates by pectin at these conditions. The complexation process is reversible so that the particles turn into soluble droplets (emulsion) again with increasing $\mathrm{pH}$. This versatility of pectin/ caseinate mixtures could be relevant especially for pharmaceutical applications in the field of microencapsulation of active compounds and drug delivery systems. In the particular case of oral administration, where the $\mathrm{pH}$ changes from acidic in the stomach to neutral at the intestine, pectin/caseinate particles would be excellent candidates.

\section{Acknowledgments}

We wish to thank CAPES (Coordenação de Aperfeiçoamento de Pessoal de Nível Superior) for the financial support of this work and CP Kelco and Kauffmann \& Co for samples supplies. We thank C.G. de Kruif and R.H. Tromp for useful discussions. We thank M.G. McPhie for a critical reading of the manuscript.

\section{References}

(1) Tolztoguzov, V.B. Functional properties of food protein and role of proteinpolysaccharide interaction. Food Hydrocol. 1991, 4, 429-468.

(2) Schmitt, C.; Sanchez, C.; Desobry-Banon, S.; Hardy, J. Structure and technofunctional properties of protein-polysaccharide complexes: a review. Crit. Rev. Food Sci. Nutr. 1998, 38 (8), 689-753. 
(3) Doublier, J. L., Garnier, C., Renard, D., \& Sanchez, C.. Protein-polysaccharide interactions. Curr. Opin. Colloid Interface Sci. 2000, 5 (3), 202-214.

(4) Turgeon, S. L.; Beaulieu, M.; Schmitt, C., \& Sanchez, C. Protein-polysaccharide interactions: phase-ordering kinetics, thermodynamic and structural aspects. Curr. Opin. Colloid Interface Sci. 2003, 8 (4-5), 401-414.

(5) Kruif, C.G. Supra-aggregates of Casein Micelles as a Prelude to Coagulation $J$. Dairy Sci. 1998, 81 (11), 3019-3028.

(6) Walstra, P. On the stability of casein micelles. Dairy Foods 1990, 73 (8), 19651979.

(7) Antonov, Yu.A.; Grinberg, V.Ya.; Tolstoguzov, V.B. Incompatibility of proteins and polysaccharides in aqueous media. Vysokomolek. Soed. 1976, 18B, 566-569 (in Russian).

(8) Amice-Quemeneur, N.; Haluk, J.P.; Hardy, J.; Kravtchenko, T.P. Influence of the acidification process on the colloidal stability of acidic milk drinks prepared from reconstituted nonfat dry milk. J. Dairy Sci. 1995, 78 (12), 2683-2690.

(9) Maroziene, A., \& de Kruif, C.G. Interaction of pectin and casein micelles. Food Hydrocol. 2000, 14 (4), 391-394.

(10) Tuinier, R.; Rolin, C.; de Kruif, C.G. Electrosorption of pectin onto casein micelles. Biomacromolecules 2002, 3 (3), 632-638.

(11) Tromp R.H., de Kruif C.G., van Eijk M., \& Rolin C.. On the mechanism of stabilization of acidified milk drinks by pectin. Food Hydrocol. 2004, 18 (4), 565572. 
(12) Pereyra, R.; Schmidt, K.A.; Wicker, L. Interaction and stabilization of acidified casein dispersions with low and high methoxyl pectins. J. Agric. Food Chem. 1997. 45 (9), 3448-3451.

(13) Matia-Merino, L.; Lau, K.; Dickinson, E. Effects of low-methoxyl amidated pectin and ionic calcium on rheology and microstructure of acid-induced sodium caseinate gels. Food Hydrocol. 2004, 18 (2), 271-281.

(14) Laemmli, U.K. Cleavage of structural proteins during the assembly of the head of bacteriophage T4. Nature 1970, 227 (5259), 680-685.

(15) Tromp, R.H.; Van De Velde, F.; Riel, J.; Paques, M. Confocal scanning light microscopy (CSLM) on mixtures of gelatine and polysaccharides. Food Res. Int. 2001, 34 (10), 931-938.

(16) Bradford, M.M. A rapid and sensitive method for the quantitation of microgram quantities of protein utilizing the principle of protein-dye binding. Anal. Biochem. 1976, 72 (7), 248-254.

(17) Panouillé, M.; Durand, D.; Nicolai, T.; Larquet, E.; Boisset, N. Aggregation and gelation of micellar casein particles. J. Colloid Interface Sci. 2005, 287, 85-93.

(18) Nelson, B.K.; Barbano, D.M. A microfiltration process to maximize removal of serum proteins from skim milk before cheese making. J. Dairy Sci. 2005, 88, 1891-1900.

(19) Tomotake, H.; Okuyama, R.; Katagiri, M.; Fuzita, M.; Yamato, M.; Ota, F. Comparison between Holstein cow's milk and Japanese-Saanen goat's milk in fatty acid composition, lipid digestibility and protein profile. Biosci. Biotechnol. Biochem. 2006, 70 (11), 2771-2774. 
(20) Grinberg, V. Ya; Tolstoguzov, V.B. Thermodynamic incompatibility of proteins and polysaccharides in solutions. Food Hydrocol. 1997, 11 (22), 145-158.

(21) Kruif, C.G.J.; Tuinier, R.; Holt, C.; Timmins, P.A.; Rollema, H.S. Physicochemical Study of $\kappa$ and $\beta$-Casein Dispersions and the Effect of CrossLinking by Transglutaminase. Langmuir 2002, 18 (12), 4885-4891.

(22) Asakura, S.; Oosawa, F. On the interaction between two bodies immersed in a solution of macromolecules. J. Chem. Phys. 1954, 22 (7), 1255-1256.

(23) Vrij, A. Polymers at interfaces and the interactions in colloidal dispersions. Pure Appl. Chem. 1976, 48 (4), 471.

(24) Aarts, D.G.A.L.; Tuinier, R.; Lekkerkerker, H.N.W. Phase behaviour of mixtures of colloidal spheres and excluded-volume polymer chains. J. Phys. Cond. Matt. 2002, 14, 7551-7561.

(25) Kar, F.; Arslan, N. Effect of temperature and concentration on viscosity of orange peel pectin solutions and intrinsic viscosity-molecular weight relationship. Carbohydr. Polymer 1999, 40 (4), 277-284.

(26) Dhont, J. K. G. An introduction to dynamics of colloids, chapter 9: Phase separation kinetics, Elsevier, Amsterdam, 1996.

(27) Bhat, S., Tuinier, R. and Schurtenberger, P., Spinodal decomposition in a food colloid-biopolymer mixture: evidence for a linear regime, J. Phys.: Condens. Matt. 2006, 18, L339.

(28) Aarts, D.G.A.L.; Dullens, R.P.A; Lekkerkerker, H.N.W. Interfacial dynamics in demixing systems with ultralow interfacial tension. New J. Phys. 2005, 7, 40. 
(29) Tuinier, R.; Dhont, J.K.G..; de Kruif, C.G. Depeltion-induced phase separation of aggregated whey protein colloids by an exocellular polysaccharide. Langmuir 2000, 16, 1497-1507.

(30) Onuki, A. Effects of shear flow and viscosity difference on phase separation. Int. J. Thermophys. 1995, 16 (2), 381-390.

(31) Lorén, N.; Hermansson, A-M. Phase separation and gel formation in kinetically trapped gelatin/maltodextrin gels. Int. J. Bio. Macro. 2000, 27 (4), 249-262.

(32) Anderson, V.J.; Jones, R.A.L. The influence of gelation on the mechanism of phase separation of a biopolymer mixture. Polymer 2001, 42 (23), 9601-9610.

(33)Butler, M.F. Mechanism and kinetics of phase separation in a gelatinymaltodextrin mixture studied by small-angle light scattering. Biomacromolecules 2002, 3 (4), 676-683.

(34) Hoog, E.H.A.; Tromp, R.H. On the phase separation kinetics of an aqueous biopolymer mixture in the presence of gelation: the effect of the quench depth and the effect of the molar mass. Colloids Surf. A: Physicochem. Eng. Aspects 2003, $213(2-3), 221-234$.

(35) Kita, R.; Kaku, T.; Kubota, K. Dobashi, T. Pinning of phase separation of aqueous solution of hydroxypropylmethylcellulose by gelation. Phys. Lett. A 1999, $259(3-4), 302-307$.

(36) Laneuville, S.I.; Sanchez, C.; Turgeon, J.; Hardy, J.; Paquin, P. in: Dickson, E. (ed.), Food Colloids - Interactions, Microstructure and Processing, The Royal Society of Chemistry: Cambridge, 2005. 
(37) Girard, M.; Sanchez, C,; Laneuville, S.; Turgeon, S.L.; Gauthier, S.F. Associative phase separation of $\beta$-lactoglobulin/pectin solutions: a kinetic study by small angle static light scattering. Colloids Surf. B Biointerface 2004, 35 (1), 15-22.

(38) Mekhloufi, G.; Sanchez, C.; Renard, D.; Guillemin, S.; Hardy, J. pH-Induced Structural Transitions during Complexation and Coacervation of $\beta$-Lactoglobulin and Acacia Gum Langmuir 2005, 21 (1), 386-394.

(39) Schmitt, C.; Sanchez, C.; Lamprecht, A.; Renard, D.; Lehr, C.M.; de Kruif, K.G.; Hardy, Study of $\beta$-lactoglobulin/acacia gum complex coacervation by diffusingwave spectroscopy and confocal scanning laser microscopy. Colloids Surf. B Biointerface 2001, 20 (3), 267-280.

(40) Sanchez, C.; Mekhloufi, G.; Schmitt, C.; Renard, D.; Robert, P.; Lehr, C.M.; Lamprecht, A. Hardy, J. Self-Assembly of $\beta$-Lactoglobulin and Acacia Gum in Aqueous Solvent: Structure and Phase-Ordering Kinetics. Langmuir 2002, 18 (26), 10323-10333.

(41) Burgess, D.J. Practical analysis of complex coacervate systems. J. Colloid Interface Sci. 1990, 140 (11), 227-238.

(42) Weinbreck, F.; de Vries, R.; Schrooyen, P.; de Kruif, C.G. Complex coacervation of whey protein and gum arabic. Biomacromolecules 2003, 4 (2), 293-303. 


\section{Legends to the Figures:}

Figure 1. Image of the SDS-PAGE gel with separated sodium caseinate fractions.

Figure 2. Phase diagram of pectin/casein mixtures at $\mathrm{pH} 7$ in $0.01 \mathrm{M}$ phosphate buffer. Diamonds, binodal points; circles, initial concentrations; square, critical point; thick full curve, binodal; thin full line, tie lines. Images show mixtures with different volumes of polymer phases for the same tie-line: upper, $75 \%$ pectin / $25 \%$ caseinate; middle, $50 \%$; lower, $25 \%$ pectin / 75\% caseinate. Bright areas correspond to pectin-rich phase.

Figure 3. Effect of temperature on the phase diagram of pectin/caseinate mixtures at $\mathrm{pH}$ 7. Samples were incubated at $45^{\circ} \mathrm{C}$ (triangles, two series of experiments), $20^{\circ} \mathrm{C}\left({ }^{\times}\right)$and $5^{\circ} \mathrm{C}\left({ }^{+}\right)$.

Figure 4. Comparison of experimental pectin-caseinate phase diagram (A) at $\mathrm{pH} 7$ with theoretical phase diagram (B) for mixtures of hard spheres plus polymer chains with excluded volume interaction ${ }^{24}$. Symbols identify experimental (squares) and a few representative theoretical (circles) binodals connected through tie-lines. The theoretical prediction is also represented in panel A by the thin curve. Asterisks identify the critical points.

Figure 5. Specific viscosity $\eta_{\mathrm{sp}}$ over pectin concentration $\mathrm{c}$ as a function of pectin concentration measured at $\mathrm{pH}$ 7. The intercept yields the intrinsic viscosity [ $\eta]$. Measurements: data points. Straight line: linear fit at low concentrations.

Figure 6. Spinodal decomposition for the 50\%/50\% pectin/caseinate sample. (a) Intensity of the Fourier transform of the fluorescence microscopy images. The open squares represent the data set of intensities taken 0.3 seconds after mixing and the stars were data taken 1.7 seconds after mixing. The insets show the real space (field of view is $400 \mu \mathrm{m}$ ) and Fourier Transforms at the indicated times. (b) Structure size $\left(1 / \mathrm{Q}_{\max }\right)$ as a function of time.

Figure 7. Time of phase separation at $\mathrm{pH} 7$ at different temperatures. Crosses, $24 \mathrm{~h}$; asterisks, $48 \mathrm{~h}$; triangles, 72h; diamonds, 96h; squares, 120h; open circles, 144h; filled circles, stable until 1 week.

Figure 8. Viscosity of a pectin/caseinate mixture (30/30 g/l) pH 7 (panel A) and incubation time required for macroscopic phase separation (panel B) as a function of temperature.

Figure 9. A) Pectin concentration in the lower and upper phases relative to initial pectin concentration in the mixture as a function of $\mathrm{pH} . \mathrm{B}, \mathrm{C}$ ) Lower phase content at different $\mathrm{pH}$ values. Panel $\mathrm{B}$ refers to the mass ratio pectin/caseinate and panel $\mathrm{C}$ refers to the total biopolymer (pectin + caseinate) concentration in the lower phase.

Figure 10. Transmission (row A) and CSLM images (rows B and C) of pectin/ caseinate mixtures (30/30 $\mathrm{g} / \mathrm{l}$ ) at different $\mathrm{pH}$ values. Rows $\mathrm{A}$ and $\mathrm{B}$ refer to images of the mixtures just after stirring. Row $\mathrm{C}$ refers to images of the lower phases after centrifugation. The scale bar for all pictures corresponds to $10 \mu \mathrm{m}$. Bright areas correspond to pectin-rich phases.

Figure 11: CLSM images of pectin/ caseinate mixtures at three different points in the phase diagram, before and after acidification. The scale bars correspond to $10 \mu \mathrm{m}$. Bright areas correspond to pectin-rich phases. 


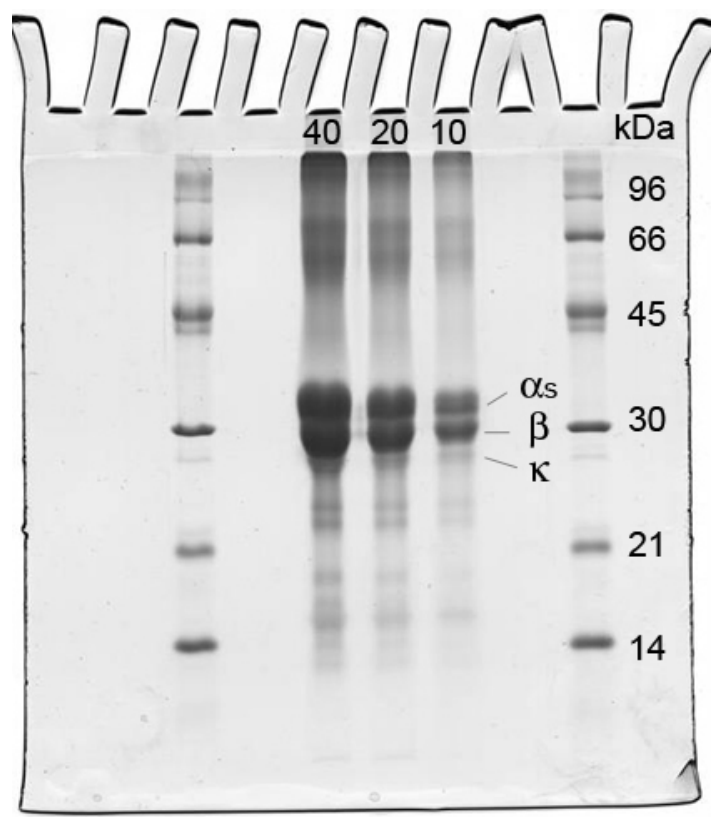

Figure 1.

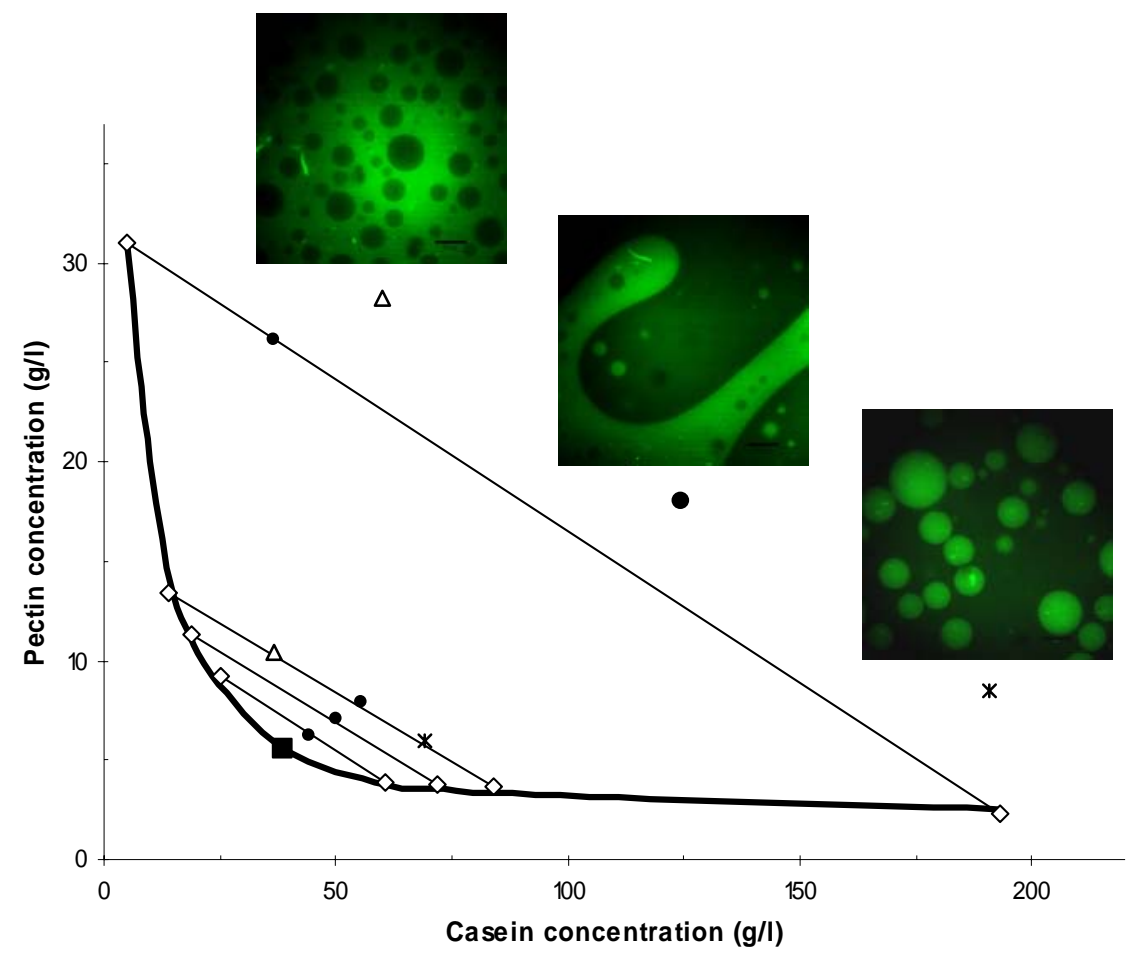

Figure 2. 


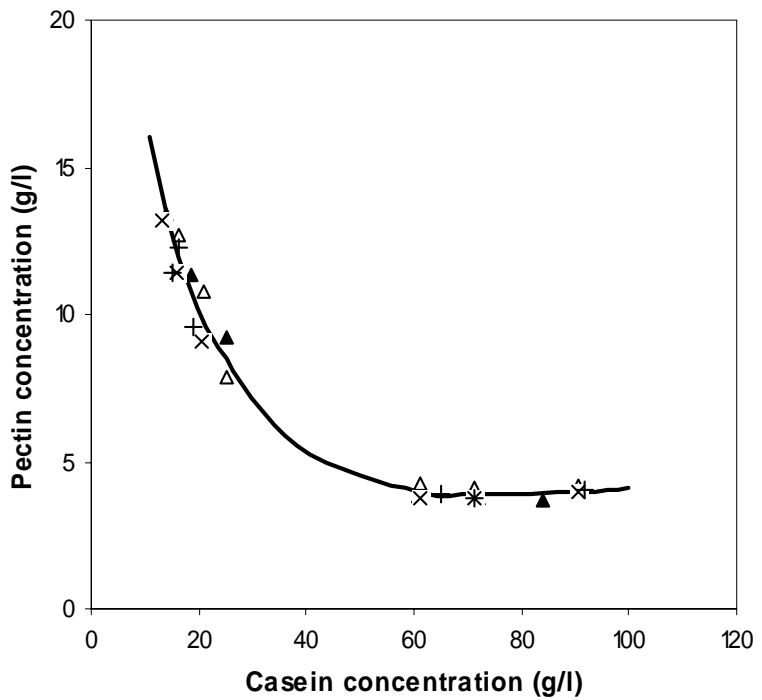

Figure 3. 

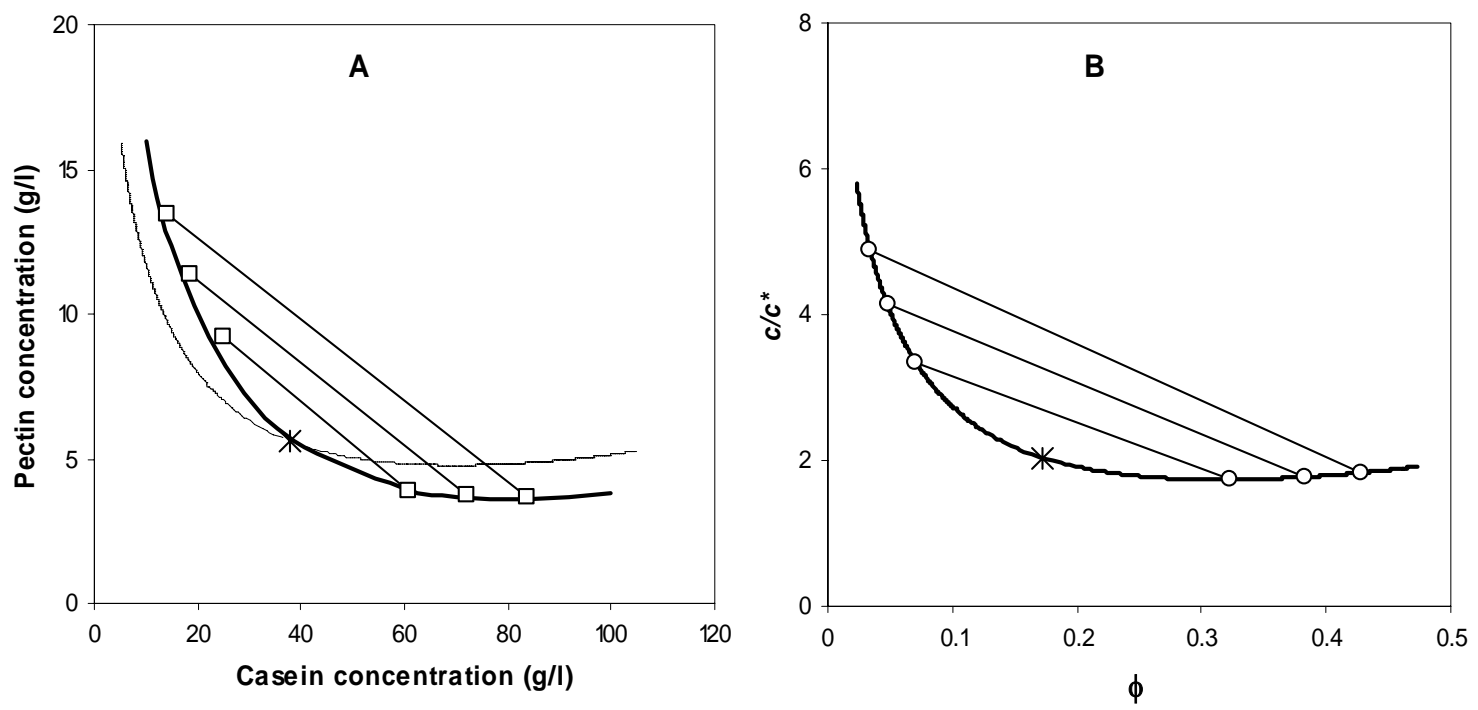

Figure 4.

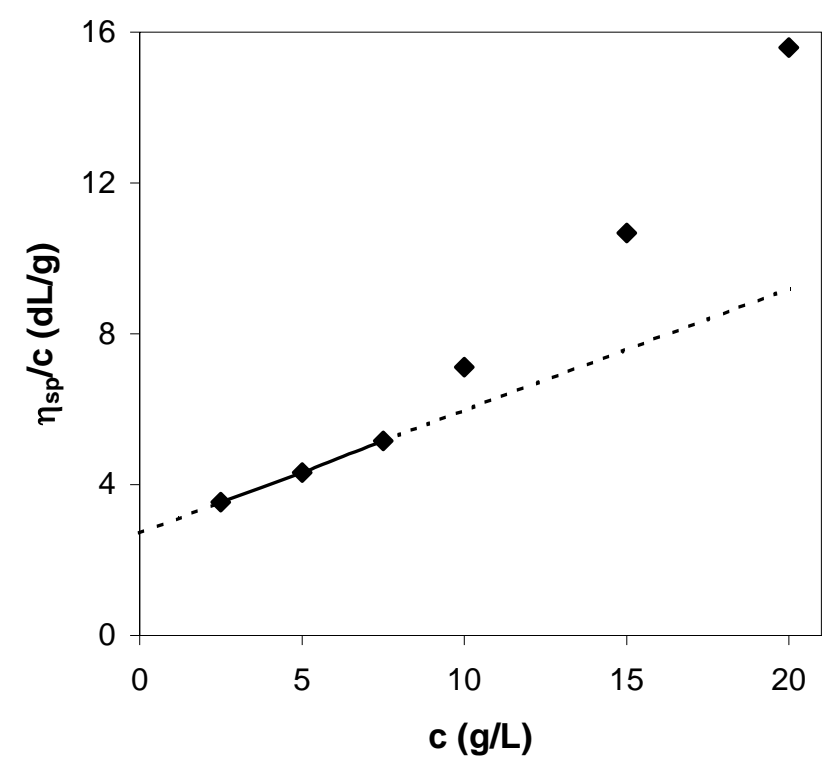

Figure 5. 


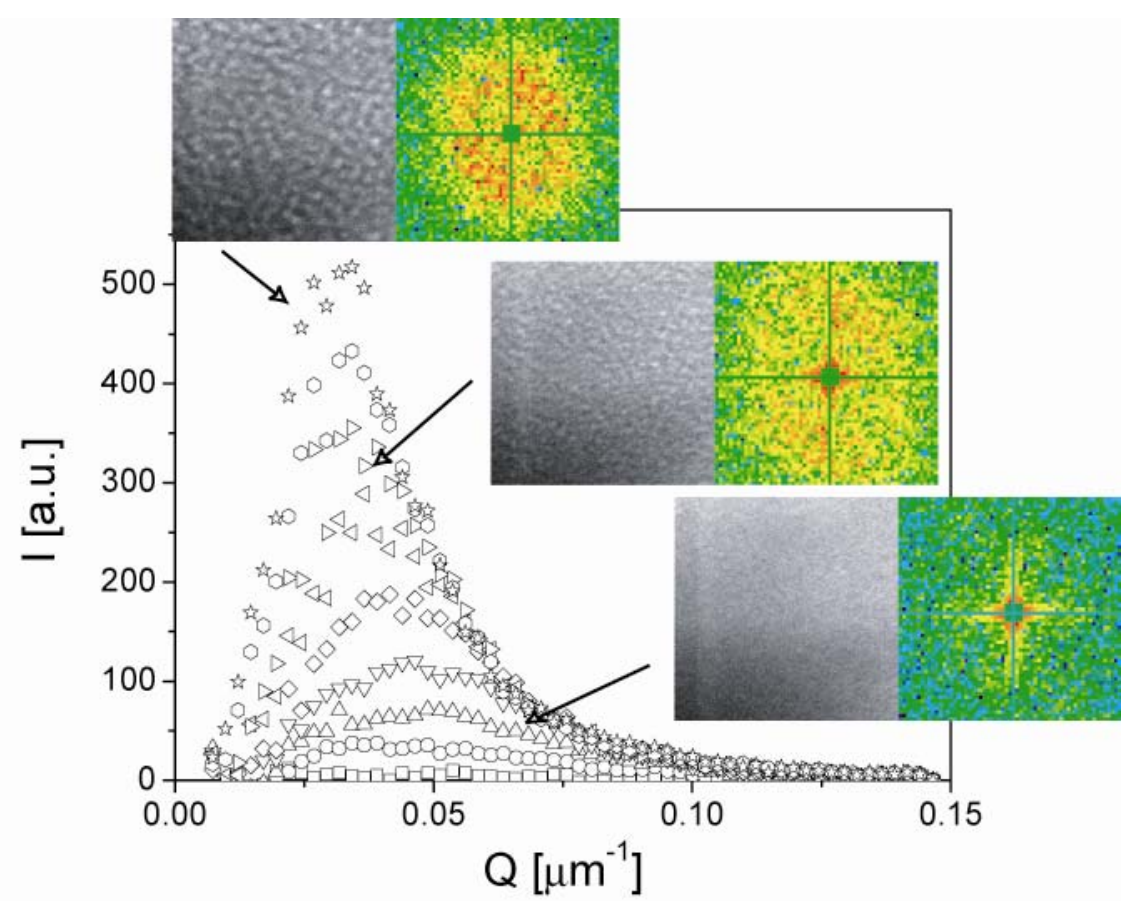

A

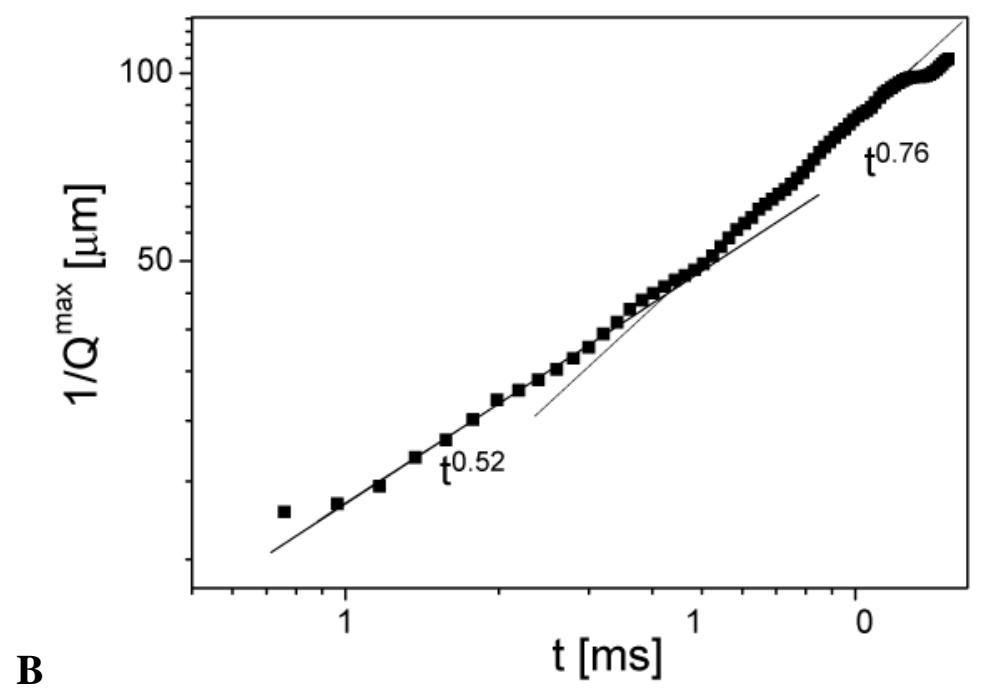

Figure 6. 

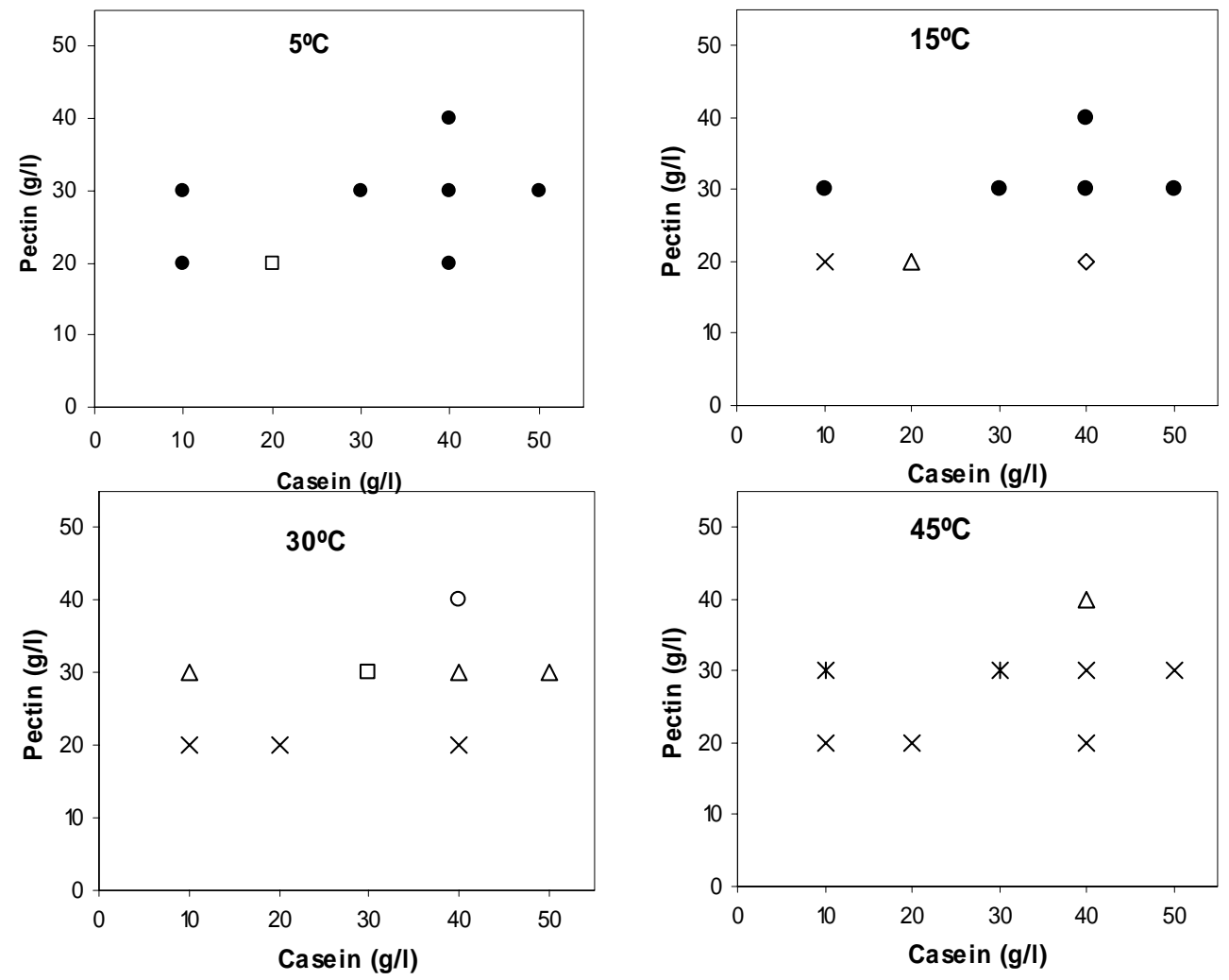

Figure 7.
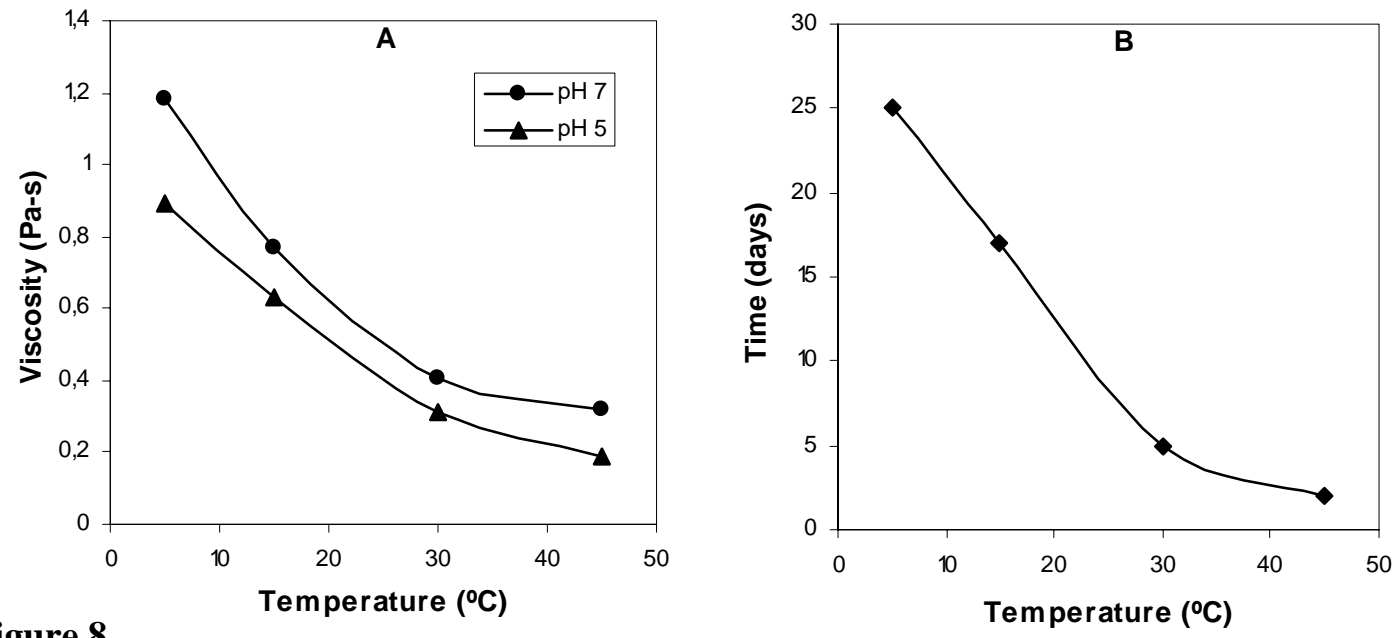

Figure 8. 

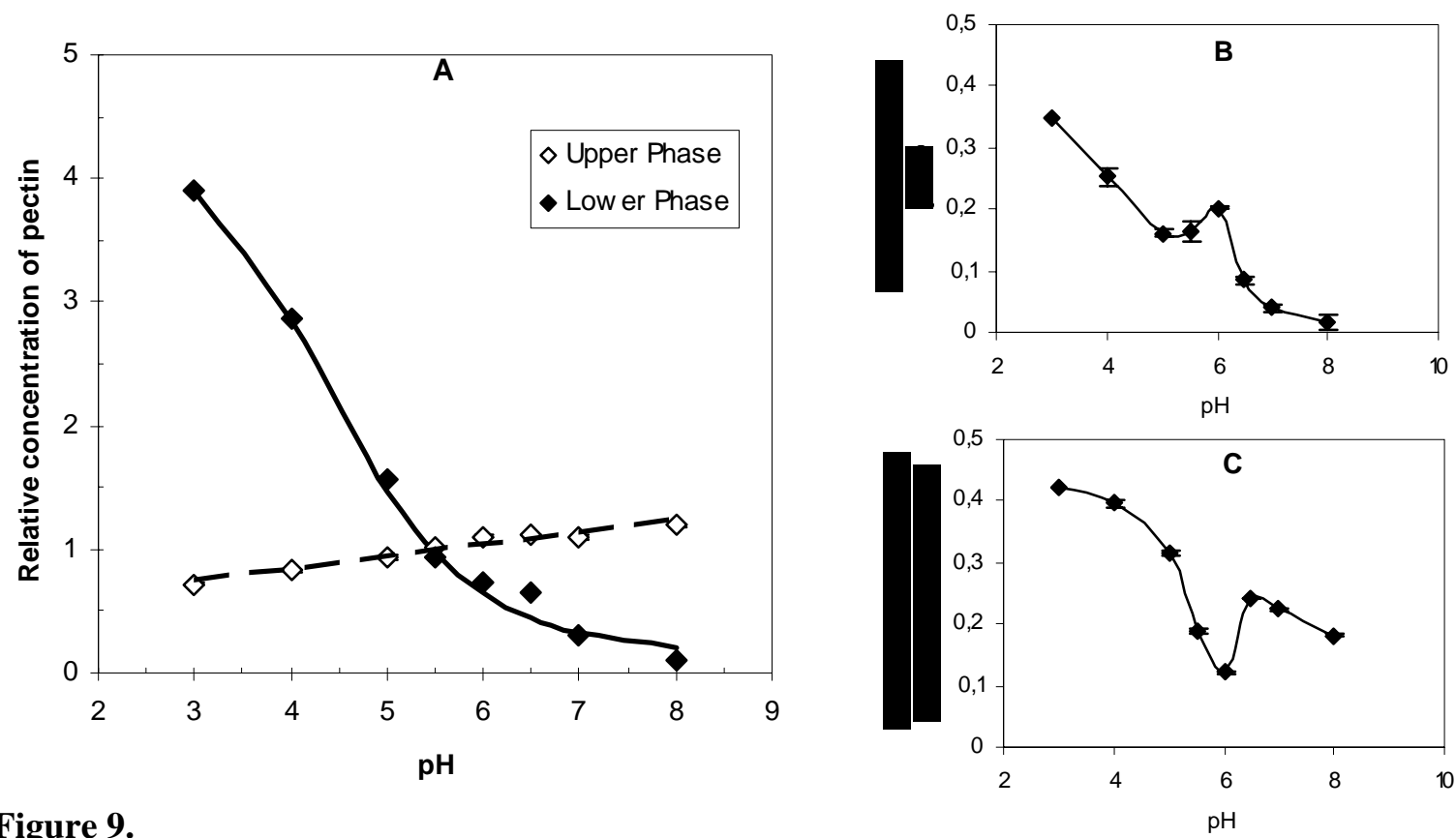

Figure 9.

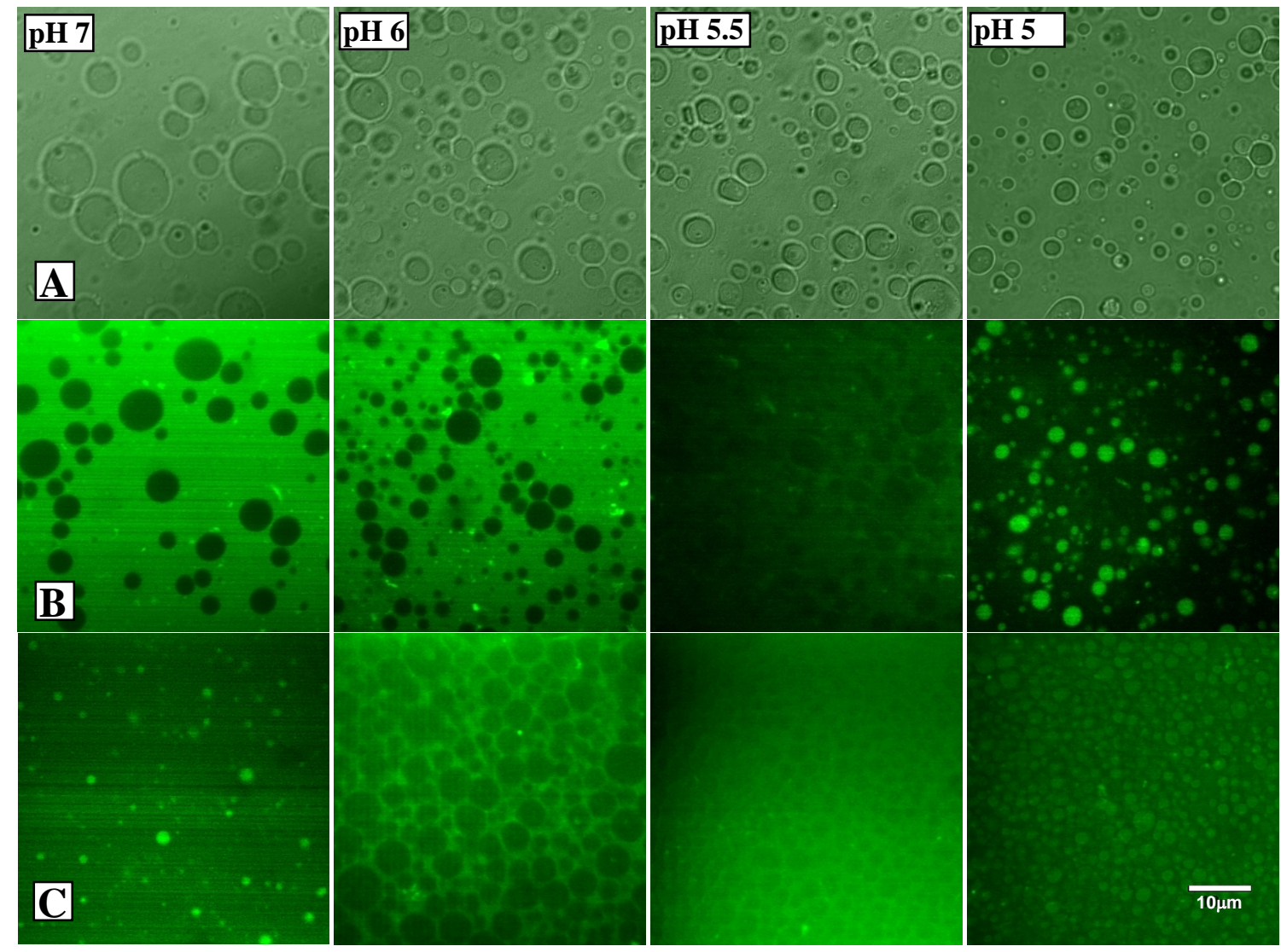

Figure 10. 


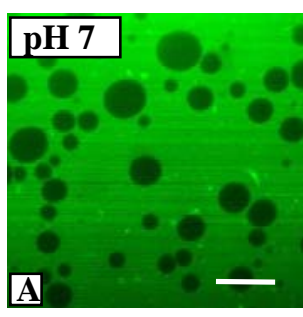

Slow

$\checkmark$ acidification

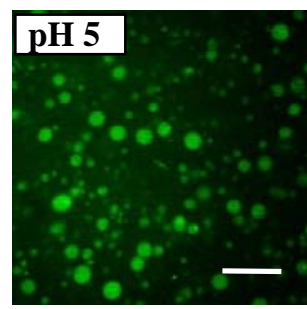

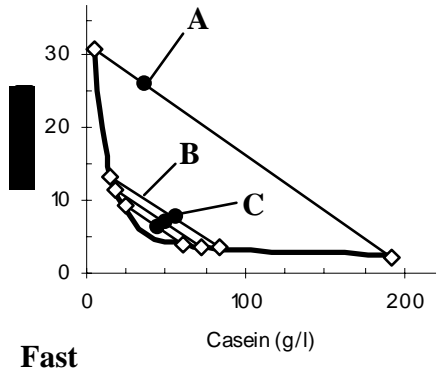

acidification

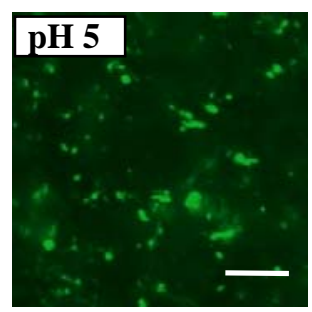

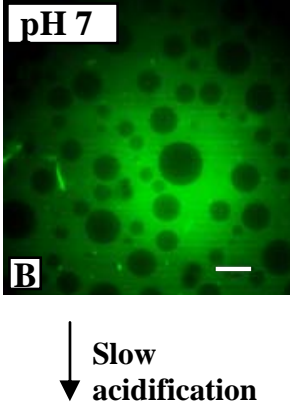

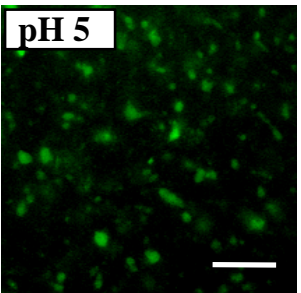

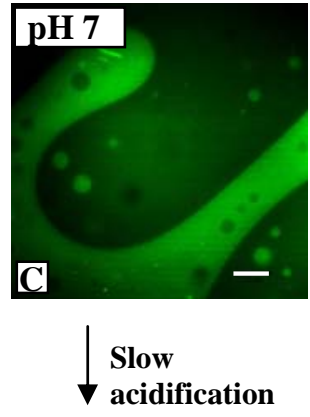

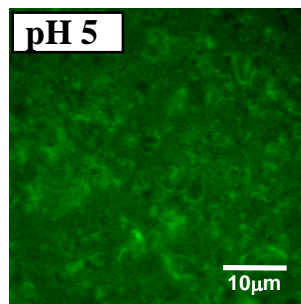

Figure 11. 